\title{
Photo detector IC for Blu-ray-Disc applications: a realization applying efficient design methodologies
}

\author{
S. Lange, T. Reich, J. Nowak, B. Dimov, M. Meister, and E. Hennig \\ Institut für Mikroelektronik- und Mechatronik- Systeme gemeinnützige GmbH, Erfurt, Germany
}

\begin{abstract}
A high-speed photo detector IC for application in Blu-ray/DVD/CD drives is presented. Bandwidths for the highest gain of $254 \mathrm{MHz}$ and $221 \mathrm{MHz}$ for $405 \mathrm{~nm}$ (Blu-ray) and $635 \mathrm{~nm}$ (DVD) wavelengths, respectively, were achieved by applying novel design methodologies. The combination of this outstanding speed performance with its low power dissipation of $192 \mathrm{~mW}$ at $5 \mathrm{~V}$ supply and the low noise power of $-72 \mathrm{dBm}$ at $300 \mathrm{MHz}$ makes it the best in literature reported optical transceiver IC for Blu-ray and Bluray/DVD/CD multi drives. Beside the excellent performance results, the usage of the novel design methodologies gave us an increased design efficiency with $25 \%$ compared to earlier similar design processes.
\end{abstract}

\section{Introduction}

In 2008 the Blu-ray Disc won the competition for the distribution media of high-definition video data. Since the data storage density of Blu-ray Discs is about one order higher compared to DVDs and in consequence much higher data rates have to be transferred, high-speed electronics is needed to read, write and rewrite the discs.

In this work a photo detector IC (PDIC) with the capability of converting high-frequency blue and red laser light into electrical signals for data reading and writing is presented. The PDIC development had to face extreme requirements of signal amplifier bandwidth with a large dynamic range at the same time. The maximum possible rotation speed of recent Blu-ray Disc drives corresponds to a $12 x$ readout speed and maximum data rates of $432 \mathrm{Mbit} / \mathrm{sec}$, which requires $3 \mathrm{~dB}$ bandwidths larger than $200 \mathrm{MHz}$ for the whole amplifier chain at all gains. In previous works maximum

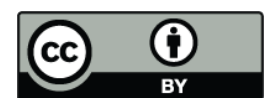

Correspondence to: S. Lange

(steffen.lange@imms.de)
$3 \mathrm{~dB}$ bandwidths for either, DVD (260 MHz in Sturm, 2005) or Blu-ray applications (204 MHz in Ko, 2009) are reported. But especially for BD/DVD multi drives a PDIC with high bandwidths for multiple wavelengths is indispensable. We combined three novel design and optimization methodologies within the design process of the presented 12x-PDIC to achieve this sophisticated goal.

The PDIC was fabricated in a $0.6 \mu \mathrm{m}$ BiCMOS process (XFAB, 2010) using integrated PIN-photodiodes with sensitivities of $0.26,0.42$ and $0.40 \mathrm{~A} / \mathrm{W}$ for wavelengths of $405 \mathrm{~nm}, 635 \mathrm{~nm}$ and $780 \mathrm{~nm}(\mathrm{CD})$, respectively. With the implemented 8 gains one can adjust the high dynamic sensitivity between 28.0 and $0.2 \mathrm{mV} / \mu \mathrm{W}$. Furthermore the device is featured by an overall power consumption of only $192 \mathrm{~mW}$ for a 10-channel system as depicted in Fig. 1.

\section{Architecture of the photo detector IC}

The schematic diagram in Fig. 1 gives an overall architecture overview of the PDIC. The PIN photodiode array is formed by 12 single diodes, divided into 4 center diodes (A-D) for beam focusing and data transfer and 8 satellite diodes (E-L) for tracking control of the laser beam. The diode array is followed by a 10-channel programmable ( 8 gains) 4-stage amplifier chain. The 6 amplifier chains A-D, RFp and RFn need to be high-speed for transmitting data up to $500 \mathrm{Mbit} / \mathrm{sec}$. The 4 channels S1-S4 operate at lower speed and the connection to the input diodes E-L is reconfigurable via a mode decoder.

Each amplifier chain consists of a current amplifier (CAp, CAn) followed by a constant transimpedance amplifier (TIA) and a buffer amplifier (VA). Applying a 2-stage current amplifier with distributed gain control ensured high-speed operation and stability for all gains within the large dynamic range. For the differential channel RFp-RFn the diode currents A-D are summed up. Gain and mode decoder can be

Published by Copernicus Publications on behalf of the URSI Landesausschuss in der Bundesrepublik Deutschland e.V. 


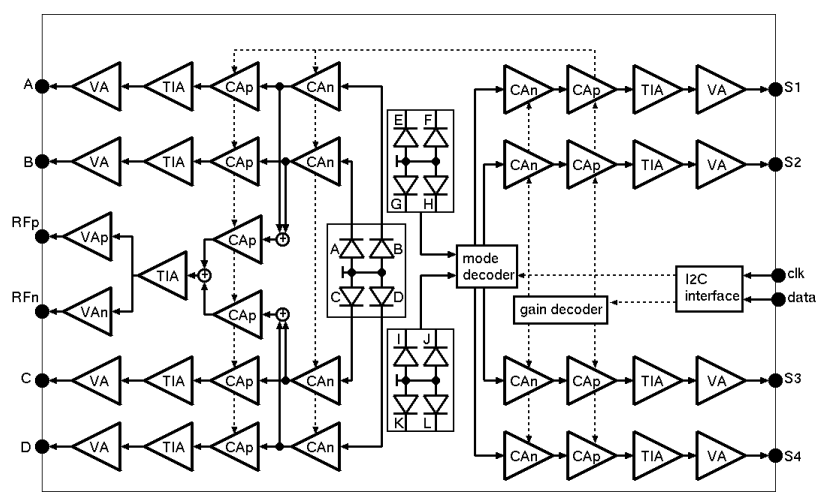

Fig. 1. Architecture overview of the complete 12x-PDIC.

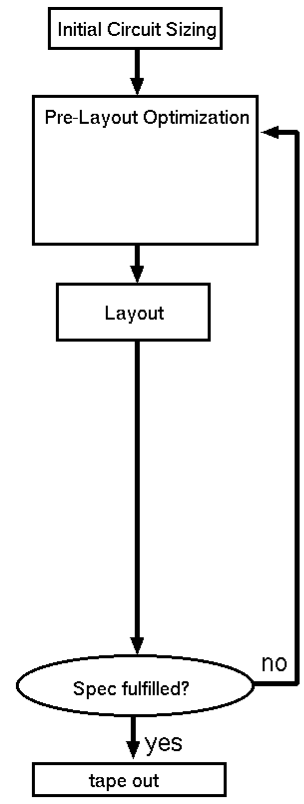

(a)

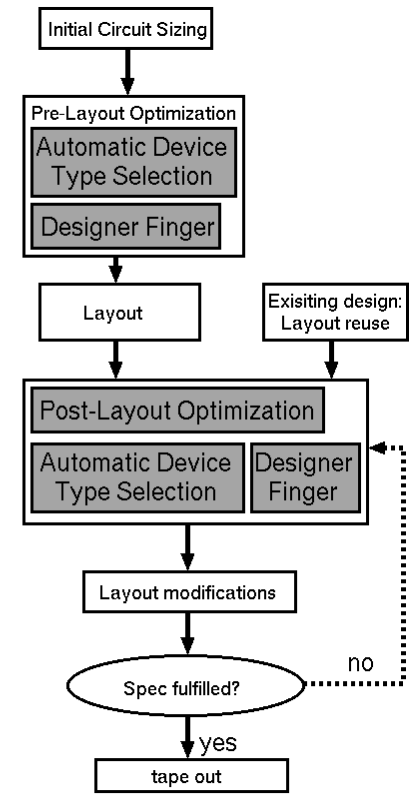

(b)

Fig. 2. Design flow within the 12x-PDIC development: (a) conventional optimization loop, (b) novel design flow applying post-layout optimization, automatic device type selection and Designer Finger.

configured via an $I^{2} C$ interface. The presented PDIC is optimized with respect to signal bandwidth, sensitivity and noise for the high-speed channels to meet the challenging requirements of Blu-ray applications.

\section{Novel methodologies for circuit optimization}

Since almost ten years our group develops successfully optical transceiver ICs starting from CD and DVD applications up to today's Blu-ray drives. Raised requirements on amplifier chain bandwidths due to higher data rates made new design methodologies indispensable. We implemented a com-

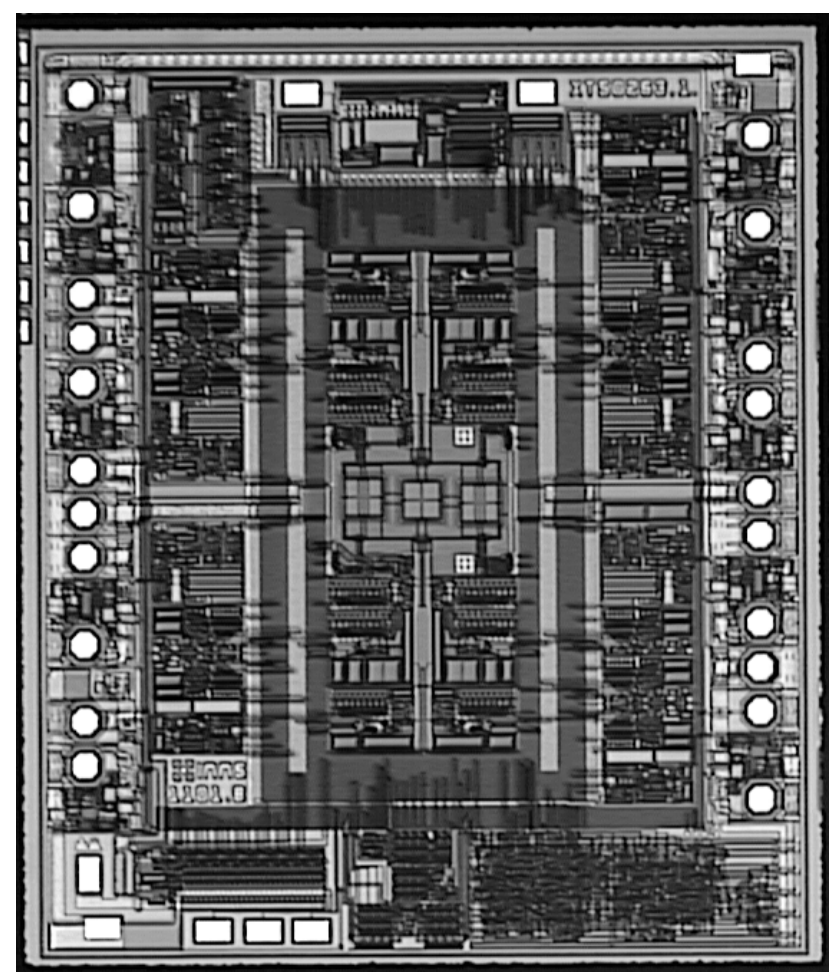

Fig. 3. Chip photograph of the PDIC.

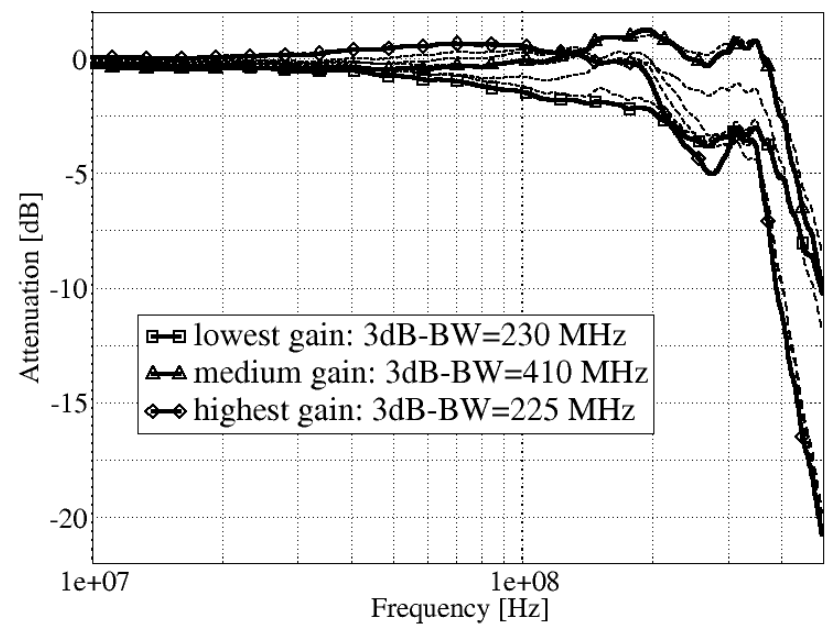

Fig. 4. Frequency response of an example high-speed channel for all 8 gains: curves for lowest, medium and highest gain are highlighted as bold lines.

bination of three novel methods within the overall design process. In Fig. 2 the difference between the conventional design and optimization flow and the novel flow including all three methodologies for efficient optimization is shown. The proposed flow in Fig. $2 b$ is suitable for complete design processes beginning from the initial topology selection and circuit sizing as well as for cost saving reuse of existing 
Table 1. Simulated and measured performances of the presented 12x-PDIC.

\begin{tabular}{|c|c|c|}
\hline Parameter & Simulated value & Measured value \\
\hline \multicolumn{3}{|l|}{ sensitivity $[\mathrm{mV} / \mu \mathrm{W}]$} \\
\hline - Blu-ray, highest/lowest gain & $28.02 / 0.22$ & $27.89 / 0.22$ \\
\hline - DVD, highest/lowest gain & - & $43.66 / 0.34$ \\
\hline \multicolumn{3}{|l|}{$3 \mathrm{~dB}$ bandwidth $[\mathrm{MHz}]$} \\
\hline - Blu-ray, medium/highest gain & $454 / 276$ & $369 / 254$ \\
\hline DVD, medium/highest gain & $454 / 276$ & $355 / 221$ \\
\hline Peaking $[\mathrm{dB}]$ & 0.27 & 0.73 \\
\hline Slew rate rise/fall $[\mathrm{V} / \mu \mathrm{s}]$ & $133 /-204$ & $145 /-135$ \\
\hline \multicolumn{3}{|l|}{ Offset voltage $[\mathrm{mV}]$} \\
\hline - Mean value (108 channels) & 1.39 & 1.0 \\
\hline - Standard deviation & - & 3.3 \\
\hline Power dissipation@ $@ \mathrm{Vdd}=5 \mathrm{~V}[\mathrm{~mW}]$ & 190 & 191.7 \\
\hline Noise power (single-ended RF channel) @ $300 \mathrm{MHz}$ [dBm] & -78 & -77 \\
\hline
\end{tabular}

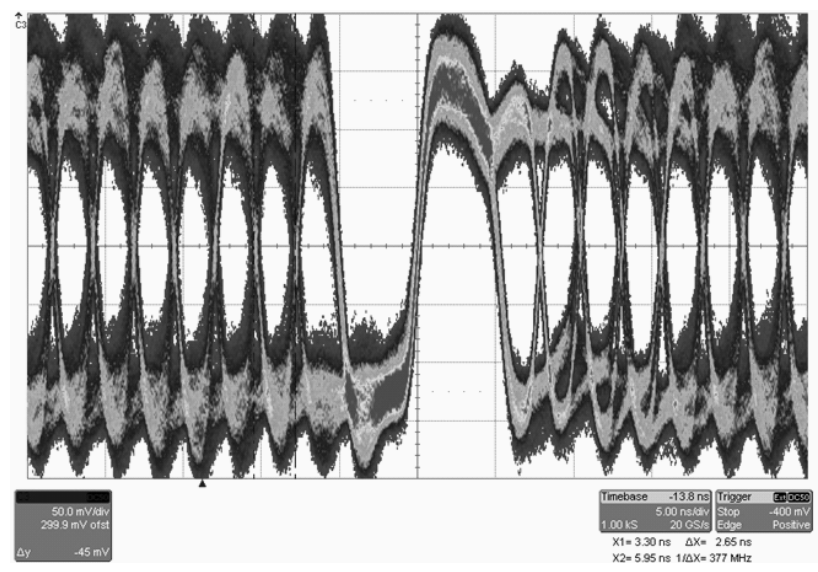

Fig. 5. Eye diagram in $12 \mathrm{x}$-mode with an eye width of $2.5 \mathrm{~ns}$ (data rate $=400 \mathrm{Mbit} / \mathrm{sec}$ ).

designs and layouts up to fulfilled specifications. The base for the design within this work ranged from existing lowspeed sub designs including layouts (e.g. VA blocks) to completely new designed blocks (e.g. CAp, CAn). Combining layout reuse with the methodologies automatic device type selection (ADTS) and post-layout optimization (PLO) for the buffer amplifier VA for the fast channels A-D, RFp and RFn was very successful in terms of circuit performance and design efficiency. The post-layout $1 \mathrm{~dB}$ bandwidth of the single block VA could be increased from $220 \mathrm{MHz}$ to $630 \mathrm{MHz}$. Furthermore, the discrepancy between pre- and post-layout performance was significantly reduced due to the intrinsic parasitics estimation of the PLO method. The layout reuse allowed a cost saving of $90 \%$ in the layout process for this particular block. Both, the PLO and ADTS methodology, are described in detail in (Reich, 2010) and (Dimov, 2008), respectively. The third novel methodology Designer Finger (Krausse, 2008) was successfully applied to the TIA. Its $1 \mathrm{~dB}$ bandwidth was increased from $260 \mathrm{MHz}$ to $385 \mathrm{MHz}$ with a remaining peaking $<1 \mathrm{~dB}$.

The presented methodologies are easy to implement into common design and optimization environments - within the presented design process Cadence 5.1 (Cadence Virtuoso, 2008) and WiCkeD analog circuit optimizer (MunEDA, 2008) were used.

\section{Experimental results}

Before PDIC characterization the laser spot characteristics was measured using a beam profiler. The full width at half maximum (FWHM) parameter was determined to $3.4 \mu \mathrm{m}$ and $6.6 \mu \mathrm{m}$ at wavelength of $405 \mathrm{~nm}$ and $635 \mathrm{~nm}$. A very good focused laser beam was used for determining the photo diode sensitivity and homogeneity of this sensitivity. The measured sensitivity of the integrated PIN diode was $0.26,0.42$ and $0.40 \mathrm{~A} / \mathrm{W}$ for wavelengths of $405 \mathrm{~nm}, 635 \mathrm{~nm}$ and $780 \mathrm{~nm}$, respectively, which fit well with the simulated values.

A chip photograph of the fabricated $12 x-P D I C$ is shown in Fig. 3. In Table 1 the simulated and measured key performances of the PDIC are listed. Intending high frequency operation, the buffer amplifier outputs have been matched with an integrated output resistance, since the downstream lowcapacitive flexible PCB is specified with a load of $2 \mathrm{pF} \| 110$ $\Omega$. Within the measurement setup the load resistance was $110 \Omega$, whereas the load capacitance was slightly higher than the one for simulations. This fact helps the reader to understand the significant discrepancy in simulated and measured performances. However, considering the strong influence of 
Table 2. Comparison of published PDIC realizations for DVD and Blu-ray drives.

\begin{tabular}{llllll}
\hline \multicolumn{1}{c}{ Parameter } & & (Sturm, 2005) & (Seidl, 2005) & (Ko, 2009) & This work \\
\hline Highest Sensitivity [mV/ $\mu \mathrm{W}]$ & Blu-ray & - & - & 12.3 & 27.9 \\
& DVD & 100 & 186 & - & 43.7 \\
\hline $3 \mathrm{~dB}$ bandwidth [MHz] (high gain) & Blu-ray & - & - & 204 & 254 \\
& DVD & 260 & 145 & - & 221 \\
\hline Offset voltage $[\mathrm{mV}]$ & & n.a. & 6.0 & n.a. & 3.3 \\
\hline Noise power & {$[\mathrm{dBm}]$} & n.a. & -66.0 & -89.8 & -77.0 \\
& @Freq. [MHz] & n.a. & 200 & 120 & 295 \\
\hline Power dissipation $[\mathrm{mW}]$ & & 300 & 163 & 140 & 192 \\
\hline Number of channels & & 10 & 10 & 6 & 10 \\
\hline
\end{tabular}

every small parasitics due to the measurement setup at high operation frequency, the achieved agreement between simulated and measured performances is superb.

In Fig. 4 the small-signal response of one high-speed channel for all gains with blue light illumination (wavelength = $405 \mathrm{~nm}$ ) is shown. A mean $3 \mathrm{~dB}$ bandwidth of $254 \mathrm{MHz}$ with $0.73 \mathrm{~dB}$ peaking was determined by measuring 25 high-speed channels on 5 PDIC devices. Furthermore, a medium gain mean $3 \mathrm{~dB}$ bandwidth of $369 \mathrm{MHz}$ with $1.28 \mathrm{~dB}$ peaking was measured. For red light (wavelength $=635 \mathrm{~nm}$ ) laser source the highest gain mean $3 \mathrm{~dB}$ bandwidth was determined to $221 \mathrm{MHz}$.

The eye diagram in Fig. 5 shows the highest gain measurement with $400 \mathrm{Mbit} / \mathrm{sec}$ pseudorandom data input, which is sufficient for the focused Blu-ray application. The limit for the realized $12 \mathrm{x}-\mathrm{PDIC}$ is bound to $500 \mathrm{Mbit} / \mathrm{sec}$ data rates, since rise and fall times of about 2 ns were determined within experiments.

The statistical distribution of the measured offset voltage with a mean value of $1 \mathrm{mV}$ and $5.8 \mathrm{mV}$ standard deviation was achieved for measuring 108 channels. By applying the internal offset compensation the standard deviation improves to $3.3 \mathrm{mV}$. A power consumption of $192 \mathrm{~mW}$ is state-of-theart among PDICs for DVD and Blu-ray drives.

The comparison with earlier publications of PDIC realizations in Table 2 substantiates the outstanding performance of the presented PDIC. It is state-of-the-art for optical transceiver ICs for fastest DVD drives with respect to bandwidth, sensitivity, noise and power dissipation. It is the best known PDIC for Blu-ray drives with the maximum $12 \mathrm{x}$ speed, since we achieved a $3 \mathrm{~dB}$ bandwidth larger than $250 \mathrm{MHz}$ for the first time. All other known publications suffer from the fact, that they are characterized for either, DVD or Blu-ray. In contrast our PDIC for Blu-ray/DVD/CD multi drives shows excellent performance for both, the fastest DVD and Blu-Ray applications.

\section{Conclusions}

A high-speed 10-channel photo detector IC for Blu-ray, DVD and CD storage drives has been presented. The implementation of novel design methodologies within the overall design flow was the base for a successful IC realization in a $0.6 \mu \mathrm{m}$ BiCMOS technology meeting the requirements of Blu-ray applications with up to $500 \mathrm{Mbit} / \mathrm{sec}$ data transfer rates. The 12x PDIC with 8 programmable gains features a power consumption of $192 \mathrm{~mW}$ and a maximum transimpedance of 214 $\mathrm{k} \Omega$ resulting in $28 \mathrm{mV} / \mu \mathrm{W}$ sensitivity. For the highest gain $3 \mathrm{~dB}$ bandwidths of $254 \mathrm{MHz}$ and $221 \mathrm{MHz}$ were achieved for wavelengths of $405 \mathrm{~nm}$ (Blu-ray) and $635 \mathrm{~nm}$ (DVD), respectively. The presented PDIC is the best known device for 12x speed Blu-ray drives and Blu-ray/DVD/CD multi drives. Furthermore its performance is state-of-the-art for DVD applications.

Acknowledgements. This work was mainly supported by the Thüringer Aufbaubank (TAB) within the research project no. 2006 VF 0046. Parts of this work were funded by the German Federal Ministry of Eduction (BMBF) within research projects HONEY (grant no. $01 \mathrm{M} 3184$ C) and SYENA (grant no. 01 M 3086).

\section{References}

Sturm, J., Leifhelm, M., Schatzmayr, H., Groiß, S., and Zimmermann, H.: Optical Receiver IC for CD/DVD/Blue-Laser Application, IEEE Journal of Solid-State Circuits, 40(7), 1406-1413, 2005.

Ko, J., Baek, K., and Kim, S.: High speed OEIC with offset drift compensation for bluray disc system, Proc. SPIE, 7219, 2009.

$\mathrm{X}-\mathrm{FAB}$ Semiconductor Foundries AG: datasheet on $0.6 \mu \mathrm{m}-$ BiCMOS process, http://www.xfab.com, 2010.

Cadence, Virtuoso Front To Back Design Environment 5.1. www. cadence.com, 2008.

MunEDA GmbH: WiCkeD Tool Suite, http://www.muneda.com, 2008. 
Cadence Design Systems: Virtuoso NeoCircuit Circuit Sizing and Optimization, http://www.cadence.com/products/custom_ic/ neocircuit/index.aspx, 2008.

Reich, T., Dimov, B., Lang, Ch., Boos, V., and Hennig, E.: A Post-Layout Optimization Method With Automatic Device Type Selection for BiCMOS Analog Circuits, IEEE 16th International Conference on Electronics, Circuits and Systems, 803806, 2010.

Dimov, B., Boos, V., Reich, T., Lang, C., Hennig, E., and Sommer, R.: A novel technique for CAD-optimization of analog circuits with bipolar transistors, Adv. Radio Sci., 7, 219-223, doi:10.5194/ars-7-219-2009, 2009.
Krausse, D., Nowak, J., Schaefer, E., Sommer, R., and Hennig, E.: Frequency Compensation by Automated Topology Modification Using Mixed Analytical and Numerical Methods for Design of Fast TIA for HDTV Applications, Proc. SM ${ }^{2}$ ACD'08, Erfurt, Deutschland, 118-126, 2008.

Seidl, C., Schatzmayr, H., Sturm, J., Groiss, S., Leifhelm, M., Spitzer, D., Schaunig, H., and Zimmermann, H.: „A Programmable OEIC for Laser Applications in the Range from $405 \mathrm{~nm}$ to $780 \mathrm{~nm}$, Proceedings of ESSCIRC, 439-442, 2005. 